\title{
Socio-Cultural Modeling through Decision-Theoretic Agents with Theory of Mind
}

\author{
David V. Pynadath and Stacy C. Marsella \\ Institute for Creative Technologies, University of Southern California \\ \{pynadath,marsella\}@ict.usc.edu
}

March 7, 2012

\begin{abstract}
PsychSim is an agent-based social simulation framework that captures a wide range of the individual and cultural differences exhibited in complex social scenarios. PsychSim takes a decision-theoretic approach to modeling Theory of Mind, giving its agents a rich space of beliefs and preferences. PsychSim also uses a unique piecewise linear representation language that allows it to reason backward from observed behavior to infer consistent parameter settings. We first applied PsychSim to the exploratory simulation of influence campaigns in the face of a heterogeneous socio-cultural arena of operations. More recently, we have used PsychSim in a range of simulation-based training systems designed to teach skills such as the correct use of language, gestures, and social norms of a foreign culture, cross-cultural negotiation, avoidance of risky behavior, and urban stabilization operations.
\end{abstract}

Keywords: social simulation, multiagent systems, theory of mind

\section{Overview}

Agent-based social simulations often seek to model socio-cultural behavior using simple models of individuals connected in large networks. However, human social and cultural behavior is also driven by complex, interrelated beliefs, attitudes and goals within individuals. A central factor in social interaction is the beliefs we have about each other, a Theory of Mind [14]. Our choice of action is influenced by how we believe others will feel and react. Whether we believe what we are told depends not only on the content of the communication but also on our model of the communicator. The central goal of this investigation is to bring such Theory of Mind capacities to the design of cross-cultural models of social interaction.

Unfortunately, traditional artificial intelligence techniques are ill-suited for modeling Theory of Mind. Representations using first-order logic are often insensitive to the distinctions among conflicting goals that people must balance in a social interaction. Resolving the ambiguity among equally possible, but unequally plausible or preferred, 
options requires a quantitative model of uncertainty and preference. Unfortunately, more quantitative frameworks, like decision theory and game theory, face their own difficulties in modeling human psychology. Game-theoretic frameworks typically rely on concepts of equilibria that people rarely achieve in unconstrained social settings. Decision-theoretic frameworks typically rely on assumptions of rationality that people frequently violate.

We have developed a social simulation framework, PsychSim [6, 10], that operationalizes existing psychological theories as boundedly rational computations to generate more plausibly human behavior. PsychSim allows a user to quickly construct a social scenario where a diverse set of entities, groups or individuals, interact and communicate. Each entity has its own preferences, relationships with other entities (e.g., friendship, hostility, authority), private beliefs, and mental models about other entities. The simulation tool generates the behavior for these entities and provides explanations of the results in terms of each entity's preferences and beliefs. The richness of the entity models allows one to easily explore the potential consequences of minor variations on the scenario.

A central aspect of PsychSim's design is that agents have fully specified decisiontheoretic models of others. Such quantitative recursive models give PsychSim a powerful mechanism to model a range of factors in a principled way. For instance, we exploit this recursive modeling to allow agents to form complex attributions about others, send messages that include the beliefs and preferences of others, and use their observations of another's behavior to influence their model of that other.

In operationalizing psychological theories within PsychSim, we have taken a strong architectural stance. We claim that decision-theoretic agents that incorporate a Theory of Mind provide a uniform, sufficient computational core for modeling the factors relevant to human social interaction. While the sufficiency of our framework remains an open question, such a strong stance yields the benefit of uniform processes and representations that cover a range of cultural factors. Our stance thus provides subsequent computational benefits, such as optimization and reuse of the core algorithms that provide the agent's decision-making and belief revision capacities.

More significantly, this uniformity begins to reveal common elements across apparently disparate psychological phenomena that typically have different methodological histories. To illustrate such common elements, we have demonstrated how a range of human psychological and social phenomena can be modeled within our framework, including wishful thinking [2], influence factors [6], childhood aggression [10] and emotion [12].

PsychSim's decision-theoretic approach to modeling Theory of Mind gives agents the same capacity that people have to possess beliefs about the beliefs and goals of other individuals and groups, both inside and outside their own culture, as well as to have those beliefs shape behavior. This capacity allows agents to form complex attributions about others, enrich the messages between agents to include the beliefs and preferences of other agents, model the impact such beliefs about others have on an agent's own behavior, model the influence that observations of another's behavior have on the agent's model of that other, etc. This rich space of beliefs and preferences allows PsychSim agents to capture a wide range of possible individual and cultural differences present in complex social scenarios. 
The complexity of such scenarios raises challenges in their authoring, simulation, and understanding. PsychSim uses a unique piecewise linear representation language to address these challenges [9]. While standard agent models work forward from model parameters to behavior, PsychSim's models are unique in that they can also work backward from observed or desired behavior, to infer the parameter changes required. In particular, PsychSim exploits linearity whenever possible to provide such bidirectional reasoning, but in a piecewise fashion so as to maintain satisfactory fidelity in modeling nonlinear phenomena.

We originally developed PsychSim for exploratory simulation of influence campaigns, with special focus on profiling contingent outcomes in the face of a heterogeneous socio-cultural arena of operations. More recently, we have applied PsychSim to a range of simulation-based training systems designed to teach skills such as the correct use of language, gestures, and social norms of a foreign culture [11], crosscultural negotiation [4], avoidance of risky behavior [5, 8], and urban stabilization operations [7]. In such training systems, students practice strategies in the safety of a simulated environment, with PsychSim agents generating behavior to simulate the responses the chosen strategies would receive in the corresponding real-world setting. We have also used PsychSim as a research tool in computational social science, to explore how a range of factors (e.g., emotions, self-deception and framing) impact beliefs and decision-making $[2,6,12]$.

\section{PsychSim}

Each PsychSim agent model starts with a representation of its current state and the Markovian process by which that state evolves over time in response to the actions performed. Thus, the overall decision problem facing a single agent maps easily into a partially observable Markov decision problem (POMDP) [3].

\subsection{State}

Each agent model includes several features representing its true state. This state consists of objective facts about the world, some of which may be hidden from the agent itself. Various PsychSim applications have included such state features as military power, economic resources, and political capital to describe the various types of resources that a person or group might have at a given point in the simulation. More subjective aspects, like moral authority, political power, and negotiation satisfaction, also correspond to state features of these same entities. Other entities represent regions or structures in the physical environment with state features like physical security and functional capacity. State features can also quantify binary relationships, such as trust, liking, and political support, that exist between two entities. We represent the overall state of the simulation at a point in time as a vector, where each component corresponds to one of these state features and has a real value in the range $[-1,1]$. 


\subsection{Actions}

Agents have a set of actions that they can choose to change the world. An action consists of an action type (e.g., offer aid, repair structure, attend rally), an agent performing the action (i.e., the actor), and possibly another agent who is the object of the action (e.g., the recipient of the aid, the structure being repaired, the organizer of the rally).

\subsection{World Dynamics}

The state of the world changes in response to the actions performed by the agents. We model these dynamics using a transition probability function to capture the possibly uncertain effects of these actions on the subsequent state. For example, repairing a structure impacts the functional capacity of that structure, as well as the economic resources of those who benefit from its output (e.g., the townspeople who get electricity from the power plant). The distribution over the change in functional capacity is a function of the capability of the actor (e.g., more skill, manpower, time, etc. on the part of the repairing agency generates a greater increase).

\subsection{Preferences}

PsychSim's decision-theoretic framework represents an agent's incentives for behavior as a reward function that maps the state of the world into a real-valued evaluation of benefit for the agent. Existing scenarios have been able to separate components of this reward function into weighted subgoals of minimizing/maximizing a state feature of a given agent. For example, agents typically have goals of maximizing their own economic resources, political power, moral authority, etc. They will also have goals looking to maximize corresponding features of their friends and allies, as well as minimizing them for their opponents. For instance, a peacekeeping force could have a goal of minimizing the resources and power of an insurgent group. These goals give the agents incentives for choosing actions that increase or decrease the relevant state features as appropriate.

While there is much similarity in the set of goals the agents have, there is great variety in their relative importance. We thus represent the overall preferences of an agent as a vector of weights, so that the product of these weights and the current state vector explicitly quantifies the degree of satisfaction that the agent receives from the world, By modifying the weights on the different goals, we can alter the motivation of the agent and, thus, its behavior in the simulation. For example, an agent that has a higher weight for maximizing its economic resources than maximizing its physical security would be more willing to still attend work despite acts of violence in the neighborhood.

\subsection{Beliefs about Others}

Sections 2.1-2.4 represent components of an underlying Markov Decision Problem of an agent's decision-making, but in general, people have only partial observability of the state of the world. Thus, they must form beliefs about that state based on their perceptions. Software agents can solve such a decision problem using existing algorithms 
to form their beliefs and then determine the action that maximizes their reward given those beliefs [3]. However, we do not expect people to conform to such optimality in their behavior. Thus, we have taken the POMDP algorithms as our starting point and modified them in a psychologically motivated manner to capture more humanlike behavior. This bounded rationality better captures the reasoning of people in the real-world, as well as providing the additional benefit of avoiding the computational complexity incurred by an assumption of perfect rationality.

The agents have only a subjective view of the world, where they form beliefs, about what they think is the state of the world. Agent A's beliefs about agent B have the same structure as the real agent B. Thus, our agent belief models follow a recursive structure, similar to previous work on game-theoretic agents [1]. Of course, the nesting of these agent models is potentially unbounded. However, although infinite nesting is required for modeling optimal behavior, people rarely use such deep models [13]. Across the PsychSim application scenarios developed so far, we have found 2-level nesting to be sufficiently rich to generate the desired behavior. Thus, the agents model each other as 1-level agents, who, in turn, model each other as 0-level agents, who do not have any beliefs. Thus, there is an inherent loss of precision (but with a gain in computational efficiency) as we move deeper into the belief structure.

For example, an agent's beliefs may include its subjective view on states of the world: I believe that our country is militarily powerful, etc. These beliefs may also include its subjective view on beliefs of other agents: I believe that the general population thinks the country is economically weak. An agent may also have a subjective view of the preferences of other agents: I believe that our leaders care more about rich people. It is important to note that we also separate an agent's subjective view of itself from the real agent. We can thus represent errors that the agent has in its view of itself (e.g., a leader believes his political power to be higher than it actually is).

\subsection{Behavior Generation}

Each agent's policy of behavior is a function that represents the process by which it selects an action or message based on its beliefs. An agent's policy allows us to model critical psychological distinctions such as reactive vs. deliberative behavior. We model each agent's real policy as a bounded lookahead procedure that seeks to maximize expected reward by simulating the behavior of the other agents and the dynamics of the world in response to the selected action/message. Each agent computes a quantitative value of each possible action given its beliefs. The agent computes the posterior probability of subsequent belief states by using the transition function to project the immediate effect of the action on its beliefs. It then projects a fixed number of steps into the future, weighing each state against its goals. Thus, the agent is seeking to maximize the expected reward of its behavior as in a POMDP. However, PsychSim's agents are only boundedly rational, given that they are constrained, both by the finite horizon of their lookahead and the possible error in their belief state. By varying the horizon for different agents, we can model entities who display different degrees of reactive vs. deliberative behavior in their thinking. 


\subsection{Model Fitting}

While PsychSim's decision-theoretic framework is well-suited to capture a person's uncertainty in beliefs and priorities among conflicting goals, the quantitative parameterization complicates the problem of constructing the models in the first place. The large parameter space encompassed by a POMDP-based model's specification of the world dynamics, observability, preferences, etc. leads to the common question, Where do the numbers come from?

We have begun to address this question by exploiting the structure that exists in typical scenarios. PsychSim uses piecewise linear functions to represent probability distributions and utility functions without sacrificing much generality. The linearity properties of our representation form the basis for a suite of algorithms that can assist in the authoring and explanation of social simulations [9]. One such algorithm addresses the complexity of the action evaluation procedure of Section 2.6. While this computational cost is prohibitive in general, the piecewise linear structure of our agent model supports an algorithm that can compile a value function into a decision tree for rapid behavior generation.

A second algorithm can take a sequence of actions and automatically generate a reward function that would generate that behavior within our agent model. This algorithm allows us to efficiently fit a PsychSim agent model to observed behavior. Our method exploits knowledge about the qualitative structure (in the form of our piecewise linearity) of a problem domain to automatically derive the correct quantitative values that would generate an observed pattern of agent behavior.

A slight variation of this model-fitting algorithm also supports analytical forward projection of PsychSim's generative models to determine the reachability of global states of interest. We have implemented algorithms that exploit this capability in exploratory simulations that identify changes in conditions (e.g., levels of trust, priorities of goals, beliefs about capabilities) that would lead to certain outcomes (e.g., acts of violence, acceptance of a ceasefire).

\section{PsychSim in Practice}

PsychSim's ability to capture a wide range of social behavior has made it a useful component in building simulations to teach students skills such as the correct use of language, gestures, and social norms of a foreign culture [11], cross-cultural negotiation [4], avoidance of risky behavior [5, 8], and urban stabilization operations [7]. In such training systems, students can execute different strategies in the safety of a simulated environment, while still seeing the consequences (through PsychSim-generated response behaviors) that their chosen strategies would receive in the real-world setting.

\subsection{Tactical Language Training System}

PsychSim agents form the basis for the Thespian system for interactive narrative [11], which in turn was used within the Tactical Language Training System (TLTS). The TLTS teaches students the rudiments of a foreign language and culture by having them 
play the role of an army sergeant conducting a civil affairs mission in a foreign town. The student interacts with virtual characters using spoken language and gestures.

One of the modeling challenges in this scenario is capturing the social norms that facilitate and constrain these conversations between the student and the agent-driven characters. Thespian used PsychSim's decision-theoretic language to build a model of several conversational norms that ensure culturally appropriate face-to-face communication: making relevant responses, following natural turn-taking patterns, and having appropriate conversational flow. The agents have explicit goals of following norms in addition to their other goals, where the relative weights can be automatically tuned using the fitting process of Section 2.7.

For example, some social interactions require the affinity between the two participants to be within a certain range. On the one hand, conversational moves like greeting and thanking can happen between almost any two characters. On the other, for asking private or potentially sensitive questions (e.g. who is the leader of the town), closer affinity is required. Thespian built a model of this conversational norm by representing the dynamics of affinity within PsychSim. For example, affinity increases as agents have successful social interactions, where successful means without violating norms. Thus, if a student or agent constantly violates norms, its affinity with other characters will decrease. The model also captures deliberate attempts to increase affinity. For example, dialogue acts such as compliments, giving offers, etc. increase the affinity between two characters (when not violating any other norms). Likewise, dialogue acts like accusations will usually reduce the affinity between two characters.

The agents have their own subjective variations of this basic model of affinity and other norm-related features to capture the individual differences among the people they represent. Given these subjective models, they can anticipate which actions may not trigger the desired responses because of insufficient affinity. They can thus reason about alternate methods of achieving their ends that require less affinity. They can also adopt a more long-term alternative strategy by taking actions that first sufficiently increase affinity to allow the desired actions. More importantly, because characters have models of each other, the agents can reason about the effect of their actions with respect to the other's model of affinity, thus accounting for the fact that its interpretation of the appropriateness of an enquiry might be different from their own.

\subsection{BiLAT}

The BiLAT game-based simulation and tutoring system provides students with an environment to practice preparing for and conducting bilateral negotiations [4]. BiLAT teaches negotiation principles (drawn from domain experts) by allowing students to conduct meetings and negotiations in a cross-cultural context. BiLAT was designed and developed through a multi-disciplinary collaboration of system designers and developers, game designers, educational researchers, instructors, and subject matter experts.

During the negotiation phase of a meeting in BiLAT, the student negotiates with a PsychSim agent that has its own goals and various negotiation moves such as making, agreeing to or rejecting various offers, accepting or rejecting the overall agreement and terminating the negotiation. The agent also has a model of the student that it uses 
to reason about what negotiation moves to make. Specifically the agent can reason about what countermoves the other negotiator (the student) can make, whether the other negotiator will likely deliver on a promised offer, and so on. It also can have a model of third parties and how the negotiation outcomes will impact those third parties. The main goal of the acting agent is to increase its own negotiation satisfaction, which is based on the perceived payoff (including costs) to the agent of the various deals. This is modeled with a nonlinearity — as the perceived payoff of a negotiation continues, new, more favorable offers will impact negotiation satisfaction less. The agent may also be concerned about the negotiation satisfaction of the other agent. These two goals, his own negotiation satisfaction and the other's negotiation satisfaction, can be weighted differently. It is possible to create a range of negotiation strategies by giving agents different weights on these goals and different kinds of negotiation moves. For example, a hardball negotiator can be modeled by giving a greater weight to the agents own negotiation strategy. The agent will make and accept offers that benefit it at first but the nonlinearity will begin to give diminishing returns to making more demands, and the agent will begin to make/accept offers that benefit the student, unless the student caves in first.

\subsection{Modeling Psychological Phenomena}

In addition to the socio-cultural phenomena described in Sections 3.1 and 3.2, we have also used PsychSim to operationalize certain psychological theories for purely research purposes. For example, we mapped several components of influence theory into decision-theoretic computations that could capture the factors determining whether people would believe a message or not [6]. We also used the decision-theoretic framework to explore how a range of factors (e.g., self-deception and framing) impact beliefs and decision-making [2]. The combination of decision theory and theory of mind provided a very natural mechanism for realizing the various dimensions of the cognitive appraisal model of human emotions [12].

\section{Conclusion}

This paper has described a subset of the socio-cultural phenomena and scenarios that we have successfully modeled with PsychSim. Obviously, these successes represents only a tiny fraction of the entire space of social behavior. However, PsychSim's successes encourage us to believe that its decision-theoretic realization of theory of mind has a broad capability to represent cross-cultural decision-making. It also encourages us that we can make even more progress without abandoning our architectural stance of taking these two components as sufficient. Furthermore, it is noteworthy that almost all of PsychSim's models have been built by non-computer-scientists. By placing our modeling tools directly in the hands of subject matter experts, we have been able to get detailed feedback about the most pressing issues relevant to the problems at hand. This catalog of issues provides us with a prioritized list of next steps that we will tackle to extend the capability and validity of PsychSim to model and simulate an even wider variety of social scenarios. 


\section{Acknowledgments}

This work was sponsored by the U.S. Army Research, Development, and Engineering Command (RDECOM), and the content does not necessarily reflect the position or the policy of the Government, and no official endorsement should be inferred.

\section{References}

[1] P. J. Gmytrasiewicz and E. H. Durfee. A rigorous, operational formalization of recursive modeling. In Proceedings of the International Conference on MultiAgent Systems, pages 125-132, 1995.

[2] J. Y. Ito, D. V. Pynadath, and S. C. Marsella. Modeling self-deception within a decision-theoretic framework. Journal of Autonomous Agents and Multiagent Systems, 20(1):3-13, 2010.

[3] L. P. Kaelbling, M. L. Littman, and A. R. Cassandra. Planning and acting in partially observable stochastic domains. Artificial Intelligence, 101:99-134, 1998.

[4] J. M. Kim, J. Randall W. Hill, P. J. Durlach, H. C. Lane, E. Forbell, M. Core, S. Marsella, D. Pynadath, and J. Hart. BiLAT: A game-based environment for practicing negotiation in a cultural context. International Journal on Artificial Intelligence in Education: Special Issue on Ill-Defined Domains, 19(3):289-308, 2009.

[5] J. Klatt, S. Marsella, and N. Krämer. Negotiations in the context of AIDS prevention: an agent-based model using theory of mind. In Proceedings of the International Conference on Intelligent Virtual Agents, 2011.

[6] S. C. Marsella, D. V. Pynadath, and S. J. Read. PsychSim: Agent-based modeling of social interactions and influence. In Proceedings of the International Conference on Cognitive Modeling, pages 243-248, 2004.

[7] R. McAlinden, A. Gordon, H. C. Lane, and D. Pynadath. UrbanSim: A gamebased simulation for counterinsurgency and stability-focused operations. In Proceedings of the AIED Workshop on Intelligent Educational Games, 2009.

[8] L. C. Miller, S. Marsella, T. Dey, P. R. Appleby, J. L. Christensen, J. Klatt, and S. J. Read. Socially optimized learning in virtual environments (SOLVE). In Proceedings of the International Conference on Interactive Digital Storytelling, 2011.

[9] D. V. Pynadath and S. C. Marsella. Fitting and compilation of multiagent models through piecewise linear functions. In Proceedings of the International Conference on Autonomous Agents and Multi Agent Systems, pages 1197-1204, 2004.

[10] D. V. Pynadath and S. C. Marsella. PsychSim: Modeling theory of mind with decision-theoretic agents. In Proceedings of the International Joint Conference on Artificial Intelligence, pages 1181-1186, 2005. 
[11] M. Si, S. C. Marsella, and D. V. Pynadath. THESPIAN: An architecture for interactive pedagogical drama. In Proceedings of the International Conference on Artificial Intelligence in Education, pages 595-602, 2005.

[12] M. Si, S. C. Marsella, and D. V. Pynadath. Modeling appraisal in theory of mind reasoning. Journal of Autonomous Agents and MultiAgent Systems, 20(1):14-31, 2010.

[13] J. Taylor, J. Carletta, and C. Mellish. Requirements for belief models in cooperative dialogue. User Modelling and User-Adapted Interaction, 6:23-68, 1996.

[14] A. Whiten, editor. Natural Theories of Mind. Basil Blackwell, Oxford, UK, 1991. 